\title{
Variabilidad morfológica y evaluación agronómica de maukas Mirabilis expansa (Ruiz \& Pav.) Standl. del norte peruano
}

\author{
Morphological variability and agronomic evaluation of maukas, Mirabilis \\ expansa (Ruiz \& Pav.) Standl. of northern Peru
}

Programa de Raíces y Tubérculos Andinos, Of. 2C-111, Universidad Nacional de Cajamarca, Avenid Atahualpa 1050, Cajamarca, Perú. Email Juan Seminario: jfseminario@yahoo.es Email Miguel A. Valderrama: mvalderrama3510@hotmail.com

\section{Juan F. Seminario y Miguel A. Valderrama}

\begin{abstract}
Resumen
Se estudiaron 40 entradas de mauka, chago o miso, Mirabilis expansa (Nyctaginaceae), para determinar la variabilidad morfológica, la distribución geográfica y el comportamiento agronómico del germoplasma, colectado principalmente en el norte peruano (entre los 2300 y 3400 m de altitud) y mantenido en la Universidad Nacional de Cajamarca ( $7^{\circ} 29^{\prime} 45^{\prime \prime} \mathrm{S}, 78^{\circ} 10^{\prime} 12^{\prime \prime} \mathrm{W}$, altitud $2670 \mathrm{~m}, 14^{\circ} \mathrm{C}$ de temperatura diaria y $670 \mathrm{~mm}$ de lluvia anual). Se usaron 17 descriptores cualitativos de alta heredabilidad y se evaluaron siete componentes de rendimiento, durante tres campañas agrícolas. El fenograma generó cinco grupos o morfotipos (coeficiente de disimilitud de 0,0). Los morfotipos I, III y V se concentran en las provincias de Hualgayoc, San Miguel, Chota y Cajamarca. El morfotipo II probablemente es endémico de la Región La Libertad. La única entrada del sur del Perú (Puno), constituyó un morfotipo independiente (IV). Seis entradas: 15, 17, 24, 25, 28 (morfotipo I) y 16 (morfotipo III), alcanzaron los más altos rendimientos $\left(46-76 \mathrm{t}^{-} \mathrm{ha}^{-1}\right)$.

Palabras clave: cultivo promisorio; diversidad genética; raíz andina, Cajamarca.

Abstract

Forty accessions of mauka, chago or miso, Mirabilis expansa (Nyctaginaceae) were studied, in order to determine the morphological variability, geographic distribution and agronomic performance of germplasm collected mainly in northern Peru (between 2300 to $3400 \mathrm{~m}$ ) and maintained at Universidad Nacional de Cajamarca ( $7^{\circ} 29^{\prime} 45^{\prime \prime} \mathrm{S}, 78^{\circ} 10^{\prime} 12^{\prime \prime} \mathrm{W}, 2670 \mathrm{~m}, 14^{\circ} \mathrm{C}$ of daily temperature and $670 \mathrm{~mm}$ of rain annually). Seventeen qualitative descriptors of high heritability were used and seven yield components for three crop years were also evaluated. The phenogram generated five groups or morphotypes (dissimilarity coefficient of 0.0 ), which implies $87.5 \%$ of duplicates in the collection. The morphotypes I, III and V are concentrated in the provinces of Hualgayoc, San Miguel, Chota and Cajamarca. The morphotype II is probably endemic to the La Libertad region. The only accession from the south of Peru (Puno) was an independent morphotype (IV). Six accessions: 15, 17, 24, 25, 28 (morphotype I) nad 16 (morphotype III) reached the highest yields (46 - 76 t. ha-1).
\end{abstract}

Keywords: promising crops; genetic diversity; Andean root crops; Cajamarca.

\section{Introducción}

Mirabilis expansa (Ruiz \& Pav.) Standl., es una Nyctaginacea tuberosa que se distribuye desde Venezuela hasta Chile al estado silvestre y cultivado (Tabla 1). Su cultivo se concentra en las zonas alto andinas de Bolivia, Ecuador y Perú, donde es conocido como "mauka", "miso" y "chago", respectivamente. Es una de las especies más relegadas dentro del grupo de raíces y tubérculos andinos, RTAs ((NRC 1989, Vivanco 1999) y existen indicios de que su cultivo en el Perú, está en riesgo debido a múltiples factores (FAO 2009). Ha sido clasificada como especie "casi amenazada", es decir próxima a satisfacer el criterio de "vulnerable". (DS. 043-2006-AG).

Mirabilis expansa es una especie promisoria como alimento humano y animal, además de otros usos potenciales. El follaje obtenido después de la cosecha, contiene hasta $4 \%$ de proteína en base fresca ( $17 \%$ en base seca), es un importante forraje para ovinos, vacunos y animales menores. Los valores de consumo, digestibilidad y conversión alimenticia en conejos (Oryctolagus cuniculus), fue similar a los valores de la mezcla de ryegras (Lolium multiflorum) y trebol (Trifolium repens) (Bazán et al. 1996).

Sin embargo, la parte principal usada como alimento humano son sus raíces reservantes. Estas, además de ser fuente de carbohidratos, contienen niveles importantes de proteína, calcio y fósforo (Franco \& Uceda 1996, Tapia et al. 2004). Por estas
Tabla 1. Distribución de Mirabilis expansa silvestre y cultivado según diferentes autores.

\begin{tabular}{l}
\hline Silvestre \\
\hline Standley (1931): \\
Venezuela: Mucurubá. \\
Ecuador: Alausí (Chimborazo), Ambato. \\
Chile: Valparaiso. \\
Perú: Sin especificar. \\
Macbride (1937): \\
Perú: Cusco (San Sebastián). \\
Junín (Tarma). \\
Lima (Chancay, Amancaes, Río Blanco). \\
López (1995): \\
Perú: La Libertad (Otuzco: Agallpampa). \\
Seminario y Valderrama (1998): \\
Perú: La Libertad (Otuzco: Cerro Cholocday). \\
Rea (1965) \\
Bolivia: Camacho, Muñecas, Bautista, Larecaja, \\
Inquisivi. \\
Rea (1982), Tapia et al. (1996) \\
Ecuador: Pichincha, Cotopaxi. \\
Vallenas (1996): \\
Perú: Puno: Limbani (prov. Sandia), Phusca (Usicayos, \\
prov. Carabaya). \\
Franco et al. (1989), Seminario y Seminario (1995). \\
Perú: Amazonas, Cajamarca, La Libertad \\
(Huamachuco, Santiago de Chuco), Ancash (Siguas). \\
Blas (2012) (Comunicación personal) \\
Perú: Huánuco. \\
\hline
\end{tabular}


bondades constituye una alternativa alimentaria para poblaciones alto andinas, en donde la ingesta de estos nutrientes es escasa.

Dentro del componente proteico de $M$. expansa se han identificado proteínas inactivadoras de ribosomas (RIPs) que muestran actividad antifúngica y antibacterial. Vivanco et al. (1999) encontraron en las raíces de mauka, dos RIPs que las denominaron ME1 (27,5 kDa) y ME2 (27 kDa), las cuales, en las pruebas in vitro, resultaron activas y de efecto aditivo contra los hongos Pythium irregulare, Fusarium oxysporum solani, Alternaria solani, Trichoderma reesei, y Trichoderma harzianum; y contra las bacterias Pseudomonas syringae, Agrobacterium tumefaciens, Agrobacterium radiobacter, Xanthomonas campestris y Erwinia carotovora. Posteriormente, Vivanco y Flores (2000) trabajando con callos y cultivo de células en suspensión de $M$. expansa, encontraron una nueva RIP que denominaron MEC $(29 \mathrm{kDa})$ cercanamente relacionada con ME1 y producida en alta proporción (20\% de la proteína soluble total). Vepachedu et al. (2003) hicieron la caracterización molecular y el estudio de la regulación post-transcripcional de la ME1. El modo de acción de ME1 contra hongos (Rhizoctonia solani, Alternaria solani, Trichoderma reeset y Candida albicans) fue estudiada por Park et al. (2002) y contra bacterias (Escherichia coli) por Vepachedu et al. (2005).

Las perspectivas de $M$. expansa son importantes y esto explica el interés de su estudio y la introducción como cultivo en otros países, particularmente en Republica Checa y Bélgica (Klaskova \& Fernández 2011).

La colección más numerosa de mauka cultivada (56 entradas) la realizó la Universidad Nacional de Cajamarca (UNC) - Perú (Valderrama \& Seminario 2004), la que incluye por lo menos siete parientes silvestres. En Ecuador, el INIAP, conserva ex situ un total de 11 entradas (Tapia et al. 2004). Los datos sobre material conservado en bancos de Bolivia no son claros (FAO 2009).

La colección de la UNC fue caracterizada con descriptores no estandarizados y se reconocieron cinco morfotipos. Asimismo,

Tabla 2. Datos pasaporte de 40 entradas de mauka (Mirabilis expansa) cultivado, mantenido por la Universidad Nacional de Cajamarca.

\begin{tabular}{|c|c|c|c|c|c|c|c|c|}
\hline Código & Localidad & Altitud & LS & LW & Caserío & Distrito & Provincia & Región \\
\hline CCHUNC001 & Yanac & 3080 & $7^{\circ} 46^{\prime} 51^{\prime \prime}$ & $77^{\circ} 57^{\prime} 56^{\prime \prime}$ & Yanac & Huamachuco & Sánchez Carrión & La Libertad \\
\hline CCHUNC002 & Peña del Gallo & 3120 & $7^{\circ} 46^{\prime} 51^{\prime \prime}$ & $77^{\circ} 57^{\prime} 56^{\prime \prime}$ & Yanac & Huamachuco & Sánchez Carrión & La Libertad \\
\hline CCHUNC003 & Yanac & 3100 & $7^{\circ} 46^{\prime} 51^{\prime \prime}$ & $77^{\circ} 57^{\prime} 56^{\prime \prime}$ & Yanac & Huamachuco & Sánchez Carrión & La Libertad \\
\hline CCHUNC005 & Laguna Sausacocha & 3200 & $7^{\circ} 47^{\prime} 17^{\prime \prime}$ & $77^{\circ} 59^{\prime} 21$ & Laguna Sausacocha & Huamachuco & Sánchez Carrión & La Libertad \\
\hline CCHUNC006 & Yanac & 3100 & $7^{\circ} 46^{\prime} 51^{\prime \prime}$ & $77^{\circ} 57^{\prime} 56$ & Yanac & Huamachuco & Sánchez Carrión & La Libertad \\
\hline CCHUNC007 & Laguna Sausacocha & 3200 & $7^{\circ} 47^{\prime} 17$ & $77^{\circ} 59^{\prime} 21^{\prime \prime}$ & Laguna Sausacocha & Huamachuco & Sánchez Carrión & La Libertad \\
\hline CCHUNC008 & Chamis & 3200 & $7^{\circ} 47^{\prime} 17^{\prime \prime}$ & $77^{\circ} 59^{\prime} 21^{\prime \prime}$ & Laguna Sausacocha & Huamachuco & Sánchez Carrión & La Libertad \\
\hline CCHUNC009 & Laguna Sausacocha & 3200 & $7^{\circ} 47^{\prime} 17^{\prime \prime}$ & $77^{\circ} 59^{\prime} 21^{\prime \prime}$ & Laguna Sausacocha & Huamachuco & Sánchez Carrión & La Libertad \\
\hline CCHUNC010 & Peña del Gallo & 3120 & $7^{\circ} 46^{\prime} 51^{\prime \prime}$ & $77^{\circ} 57^{\prime} 56^{\prime \prime}$ & Yanac & Huamachuco & Sánchez Carrión & La Libertad \\
\hline CCHUNC011 & Peña del Gallo & 3120 & $7^{\circ} 46^{\prime} 51^{\prime \prime}$ & $77^{\circ} 57^{\prime} 56^{\prime \prime}$ & Peña del Gallo & Huamachuco & Sánchez Carrión & La Libertad \\
\hline CCHUNC012 & El Cedro & 2855 & $6^{\circ} 58^{\prime} 25^{\prime \prime}$ & $78^{\circ} 51^{\prime} 18^{\prime \prime}$ & El Cedro & Calquis & San Miguel & Cajamarca \\
\hline CCHUNC013 & El Pozo & 2855 & $6^{\circ} 58^{\prime} 25^{\prime \prime}$ & $78^{\circ} 51^{\prime} 18^{\prime \prime}$ & El Cdero & Calquis & San Miguel & Cajamarca \\
\hline CCHUNC014 & Nitisuyo Alto & 3000 & $6^{\circ} 59^{\prime} 57^{\prime \prime}$ & $78^{\circ} 53^{\prime} 58^{\prime \prime}$ & Nitisuyo & San Miguel & San Miguel & Cajamarca \\
\hline CCHUNC015 & Lucmacucho & 3100 & $6^{\circ} 37^{\prime} 26^{\prime \prime}$ & $78^{\circ} 31^{\prime} 21^{\prime \prime}$ & Samangay & Bambamrca & Hualgayoc & Cajamarca \\
\hline CCHUNC016 & Huayllapampa Baja & 3400 & $7^{\circ} 12^{\prime} 26^{\prime \prime}$ & $78^{\circ} 30^{\prime} 36^{\prime \prime}$ & Huayllapampa & Cajamarca & Cajamarca & Cajamarca \\
\hline CCHUNC017 & Llapa & 2928 & $6^{\circ} 58^{\prime} 38^{\prime \prime}$ & $78^{\circ} 48^{\prime} 48^{\prime \prime}$ & Llapa & Llapa & San Miguel & Cajamarca \\
\hline CCHUNC019 & Laguna Sausacocha & 3200 & $7^{\circ} 47^{\prime} 17^{\prime \prime}$ & $77^{\circ} 59^{\prime} 21^{\prime \prime}$ & Laguna Sausacocha & Huamachuco & SanchezCarrion & La Libertad \\
\hline CCHUNC020 & Chucmar & 2500 & $6^{\circ} 23^{\prime} 15^{\prime \prime}$ & $78^{\circ} 35^{\prime} 55^{\prime \prime}$ & Chucmar & Tacabamba & Chota & Cajamarca \\
\hline CCHUNC021 & Cañafisto & 3100 & $6^{\circ} 33^{\prime} 58^{\prime \prime}$ & $78^{\circ} 35^{\prime} 52^{\prime \prime}$ & Cañafisto & Chota & Chota & Cajamarca \\
\hline CCHUNC023 & Paccha Grande Alta & 2800 & $7^{\circ} 11^{\prime} 54^{\prime \prime}$ & $78^{\circ} 29^{\prime} 44^{\prime \prime}$ & Paccha & Cajamarca & Cajamarca & Cajamarca \\
\hline CCHUNC024 & La Collpa & 2750 & $6^{\circ} 40^{\prime} 03^{\prime \prime}$ & $78^{\circ} 44^{\prime} 13$ & La Collpa & Chugur & Hualgayoc & Cajamarca \\
\hline CCHUNC025 & Sabana & 3150 & $6^{\circ} 52^{\prime} 13^{\prime \prime}$ & $78^{\circ} 48^{\prime} 12^{\prime \prime}$ & Sabana & Llapa & San Miguel & Cajamarca \\
\hline CCHUNC026 & El Cedro & 2855 & $6^{\circ} 58^{\prime} 25^{\prime \prime}$ & $78^{\circ} 51^{\prime} 18^{\prime \prime}$ & El Cedro & Calquis & San Miguel & Cajamarca \\
\hline CCHUNC027 & Namora & 2300 & $7^{\circ} 12^{\prime} 01^{\prime \prime}$ & $78^{\circ} 19^{\prime} 34^{\prime \prime}$ & Namora & Namora & Cajamarca & Cajamarca \\
\hline CCHUNC028 & Sn.SilvestreCochan & 2950 & 6059'36" & $78^{\circ} 45^{\prime} 52^{\prime \prime}$ & Sn.SilvestreCochan & Sn.Silvestre & SanMiguel & Cajamarca \\
\hline CHAUNC029 & Sn.SilvestreCochan & 2950 & $6^{\circ} 59^{\prime} 36^{\prime \prime}$ & $78^{\circ} 45^{\prime} 52^{\prime \prime}$ & Sn.SilvestreCochan & Sn Silvestre & San Miguel & Cajamarca \\
\hline CCHUNC030 & Sn.SilvestreCochan & 2950 & $6^{\circ} 59^{\prime} 36^{\prime \prime}$ & $78^{\circ} 45^{\prime} 52^{\prime \prime}$ & Sn Silvestre & Sn.Silvestre & San Miguel & Cajamarca \\
\hline CCHUNC031 & Nitisuyo Alto & 3000 & $6^{\circ} 59^{\prime} 57^{\prime \prime}$ & $78^{\circ} 53^{\prime} 58^{\prime \prime}$ & Nitisuyo & San Miguel & San Miguel & Cajamarca \\
\hline CCHUNC036 & Chaquil & 2515 & $6^{\circ} 45^{\prime} 45^{\prime \prime}$ & $78^{\circ} 31^{\prime} 21^{\prime \prime}$ & Llaucan & Bambamarca & Hualgayoc & Cajamarca \\
\hline CCHUNC037 & Llaucan & 2500 & $6^{\circ} 44^{\prime} 21^{\prime \prime}$ & $78^{\circ} 31^{\prime} 21^{\prime \prime}$ & Llaucan & Bambamarca & Hualgayoc & Cajamarca \\
\hline CCHUNC038 & Mayhuasi & 2600 & $6^{\circ} 43^{\prime} 06^{\prime \prime}$ & $78^{\circ} 31^{\prime} 21^{\prime \prime}$ & Mayhuasi & Bambamarca & Hualgayoc & Cajamarca \\
\hline CCHUNC039 & Curgos & 3225 & $7^{\circ} 51^{\prime} 02^{\prime \prime}$ & $77^{\circ} 56^{\prime} 18^{\prime \prime}$ & Curgos & Curgos & Sanchez Carrion & La Libertad \\
\hline CCHUNC040 & Quillimbash & 2850 & $6^{\circ} 54^{\prime} 53^{\prime \prime}$ & $78^{\circ} 10^{\prime} 45^{\prime \prime}$ & Quillimbash & Celendin & Celendin & Cajamarca \\
\hline CCHUNC042 & Limbani & 3000 & $14^{\circ} 06^{\prime} 35^{\prime \prime}$ & $69^{\circ} 36^{\prime} 55^{\prime \prime}$ & Limbani & Limbani & Sandia & Puno \\
\hline CCHUNC043 & Huañambra & 2780 & $6^{\circ} 53^{\prime} 5$ & $78^{\circ} 9^{\prime} 54^{\prime \prime}$ & Huañambra & Celendin & Celendin & Cajamarca \\
\hline CCHUNC044 & Ciudad Universitaria & 2725 & $7^{\circ} 10^{\prime} 00^{\prime \prime}$ & $78^{\circ} 30^{\prime} 00^{\prime \prime}$ & Cajamarca & Cajamarca & Cajamarca & Cajamarca \\
\hline CCHUNC045 & Cochapampa & 2950 & $6^{\circ} 54^{\prime} 52^{\prime \prime}$ & $78^{\circ} 15^{\prime} 18^{\prime \prime}$ & Cochapampa & Sorochuco & Celendin & Cajamarca \\
\hline CCHUNC046 & Tacamache & 2850 & $6^{\circ} 40^{\prime} 46^{\prime \prime}$ & $78^{\circ} 45^{\prime} 06^{\prime \prime}$ & Tacamache & Chugur & Hualgayoc & Cajamarca \\
\hline CCHUNC047 & Liguñac & 2500 & $6^{\circ} 52^{\prime} 31^{\prime \prime}$ & $78^{\circ} 41^{\prime} 57^{\prime \prime}$ & Liguñac & Sócota & Cutervo & Cajamarca \\
\hline CCHUNC048 & Llaucan & 2400 & $6^{\circ} 44^{\prime} 20^{\prime \prime}$ & $78^{\circ} 01^{\prime} 11^{\prime \prime}$ & Llaucan & Bambamarca & Hualgayoc & Cajamarca \\
\hline
\end{tabular}


un lote de 36 entradas de esta misma colección (incluyendo cuatro silvestre) fue caracterizada molecularmente por Chia et al. (2006) y se identificaron cuatro morfotipos, pero con alta divergencia, respecto a la composición de mismos.

La información sobre evaluación agronómica de los materiales genéticos de mauka es escasa. Sólo existen informes preliminares de los aspectos productivos. En este sentido, es necesario evaluar los materiales mantenidos ex situ con el propósito de seleccionar los mejores con fines de uso

Con los antecedentes mencionados, el propósito de la investigación fue determinar la variabilidad morfológica, mediante la aplicación de descriptores morfológicos estandarizados, analizar la distribución geográfica y evaluar el comportamiento agronómico de la colección de mauka del Programa de Raíces y Tubérculos Andinos de la UNC, que procede principalmente del norte peruano e incluye una entrada de Puno.

\section{Material y métodos}

Se usaron 40 entradas de Mirabilis expansa cultivada, que mantiene el Programa de Raíces y Tubérculos Andinos de la UNC. (PRTA-UNC). Veintisiete entradas proceden de la Región Cajamarca (2300 a $3400 \mathrm{~m}$ de altitud), colectadas en las provincias de San Miguel (10), Hualgayoc (7), Cajamarca (4), Celendín (3), Chota (2) y Cutervo (1). Doce entradas proceden de la región La Libertad, colectadas en la provincia de Sánchez Carrión. La entrada 42 procede de Limbani, provincia de Sandia, Región Puno. Caso especial constituye la entrada 44 que fue detectada e incorporada a la colección, por sus características morfológicas especiales, desde una población de plantas provenientes de semilla, dentro del banco de germoplasma. Los datos pasaporte del germoplasma evaluado se detallan en la Tabla 2.

El germoplasma se sembró y evaluó durante las tres campañas agrícolas, en el campo experimental del PRTA-UNC. El sitio se ubica a $7^{\circ} 29^{\prime} 45^{\prime}$ 'S, 78 $10^{\circ} 12^{\prime \prime W}$ y $2670 \mathrm{~m}$, dentro de la formación Bosque Seco Montano Bajo (Quechua) y registra $14^{\circ} \mathrm{C}$ de temperatura diaria y $670 \mathrm{~mm}$ de lluvia anual. Se sembró un surco por cada entrada, con brotes basales de tallo, a distancias de $0,80 \mathrm{~m}$ entre surcos y $0,60 \mathrm{~m}$ entre plantas. Se aplicaron $5 \mathrm{t} / \mathrm{ha}$ de humus de lombriz y se hicieron las labores de mantenimiento necesarias para el cultivo.

La caracterización y el análisis de agrupamiento se realizaron mediante una lista de 17 descriptores cualitativos (Tabla 3), de alta capacidad discriminatoria (cuatro de tallo, cinco de hoja, cinco de flor y tres de raíz). Esta lista fue probada y estandarizada el 2000 por especialistas de Ecuador, Bolivia y Perú. Los datos de hoja se tomaron a los seis meses después de la siembra (inicio de floración) y los de colino y raíz se tomaron al momento de cosecha (11 meses). Los colores fueron calificados mediante la RHS Colour Chart (1995). Con los datos de campo, se construyó una matriz básica de datos (MBD) y se realizó el análisis multivariado el cual comprendió (1) el análisis de agrupamiento produciendo un fenograma generado por los métodos UPGMA (unweighted pair-group method aritmetic average) y SAHN (sequential, agglomerative, hierarchical, nonoverlapping) - clustering; (2) el análisis de componentes-principales (CP), método de ordenación que permite demostrar el valor discriminatorio de los caracteres, con respecto a las entradas (Crici 1983, Hidalgo 2003). En ambos casos se usó el programa NTSYS 2.1.
Tabla 3. Descriptores morfológicos para mauka, Mirabilis expansa (R.\&P.) Standley.

1. Color de follaje

1 Verde amarillento $144 \mathrm{~A}$

2 Verde amarillo 146C, 146A

3 Verde purpúreo

2. Color principal del tallo aéreo

1 Verde amarillo 144C, 145A

2 Verde $143 \mathrm{C}$

3 Rojo purpúreo 59B, 60A

3. Color secundario del tallo aéreo

0 Ausente

1 Presente

4. Color de tallo subterráneo

1 Blanco

2 Amarillo claro 13B

3 Naranja grisáceo 165B

5. Color predominante del haz de la hoja

1 Verde amarillento $145 \mathrm{~A}$

2 Verde amarillo $146 \mathrm{~B}$

3 Verde 146 A

6. Color secundario del haz de la hoja

0 Ausente

1 Presente

7. Forma de lámina

1 Ovada

2 Cordada

8. Base de lámina

1 Cordada

2 Cuneada

3 Subcordada

9. Apice de lámina

1 Estrechamente agudo

2 Medianamente agudo

3 Ampliamente agudo

10. Color de botón floral

1 Amarillo 4A,5B

2 Pardo amarillento 163B

3 Rojo púrpura $72 \mathrm{~B}$

4 Rojo púrpura $71 \mathrm{~A}$

11. Color de las brácteas del involucro

1 Verde $146 \mathrm{C}$

12. Color de Perigonio

3 Púrpura verdoso

1 Blanco 155D

2 Blanco grisáceo 156D

3 Púrpura claro 75B

4 Púrpura 78A

13. Color venas de lóbulos del perigonio

1 Amarillo 4A

2 Pardo amarillento 163B

3 Rojo púrpura 71A

14. Color Estigma

1 Blanco amarillento 158D

2 Amarillo anaranjado 20C

3 Púrpura claro 75D

4 Púrpura 75A

15. Color externo de la corteza de la RR

1 Blanco 155D

2 Pardo amarillento 161C

3 Gris anaranjado claro $164 \mathrm{C}$

4 Gris anaranjado 164 A, 165B

16. Color de la corteza de raíz al raspado

1 Blanco 155D

2 Amarillo $12 \mathrm{C}$

3 Amarillo anaranjado 14B, 13A

17. Color de la pulpa de la raíz reservante

1 Blanco 155D

2 Blanco anaranjado 159C

3 Amarillo anaranjado 19C, 20C 


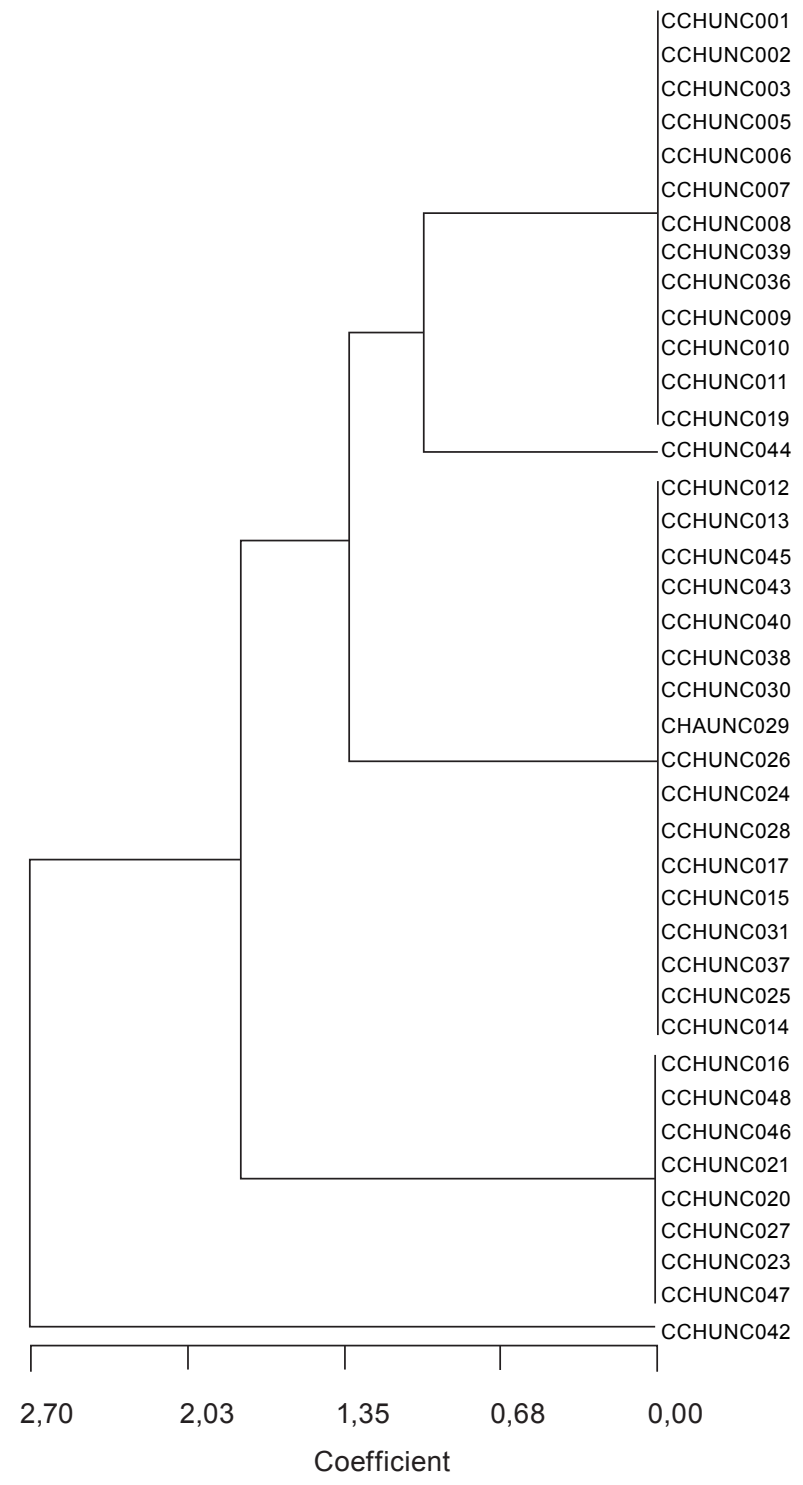

Figura 1. Fenograma (método UPGMA) que agrupa 40 entradas de mauka, Mirabilis expansa, del norte peruano, según 17 descriptores morfológicos.
El análisis de la distribución geográfica del germoplasma se hizo tomando en cuenta, las coordenadas geográficas, las altitudes, las regiones y las cuencas de los sitios de colecta. Mediante el programa DIVA-GIS 3.0 se señalaron los sitios de colecta y se hizo la distribución geográfica de las entradas agrupadas en morfotipos.

Se evaluaron siete componentes del rendimiento agronómico, en cinco plantas por entrada y en tres campańas agrícolas (2005 - 2007): Altura de planta $(\mathrm{cm})$, número de raíces por planta, peso de raíces por planta $(\mathrm{kg})$, rendimiento de raíces $\left(\mathrm{t} . \mathrm{ha}^{-1}\right)$, largo y diámetro de raíces $(\mathrm{cm})$ y peso de follaje $(\mathrm{kg})$. Los datos fueron ingresados en una hoja de cálculo de Excel, para el análisis correspondiente.

\section{Resultados}

Variabilidad morfológica. Al coeficiente de distancia de 0,0 (máxima similitud), el fenograma (Fig.1) muestra que la variabilidad morfológica de la colección de mauka, consta de cinco grupos o morfotipos (el 87,5\% de las entradas serían duplicados). Dos grandes grupos, el M-I y M-II con 18 y 12 entradas, respectivamente, reúnen el $75 \%$ de las entradas evaluadas. El coeficiente de distancia entre ambos es bajo (20\%). Esto quiere decir que estos grupos están cercanamente relacionados fenotípicamente. La entrada 42 (grupo M-IV), procedente de Puno, registró el coeficiente de distancia más alto. Es decir, es un morfotipo único y diferente a los del norte. Lo antedicho, tiene mayor sentido si tomamos como referencia el coeficiente de distancia de 1,35 (Fig. 1). Bajo esta premisa, el fenograma presenta tres grandes grupos: El primero, formado por M-II, M-I y M-V, el segundo por M-III y el tercero por M-IV. Por otro lado, la entrada 44 constituye un morfotipo aparte (M-V) y presenta características morfológicas de los grupos M-I y M-II.

Los cinco morfotipos identificados muestran características fenotípicas de fácil identificación en campo y sus principales diferencias se encuentran en el color del tallo, forma de hoja, color de flor y color de raíz. También es evidente que la mayor parte del germoplasma pertenece a los morfotipos I y II (Tabla 4).

Tabla 4. Descripción básica de los morfotipos y agrupamiento de las entradas de mauka (Mirabilis expansa) mantenidos por la Universidad Nacional de Cajamarca.

\begin{tabular}{|c|c|c|c|c|c|c|}
\hline & & \multicolumn{5}{|c|}{ Morfotipos } \\
\hline & & I & II & III & IV & V \\
\hline \multicolumn{2}{|l|}{ Color tallo } & Rojo purpúreo & Verde & Verde amarillo & Verde & Verde \\
\hline \multirow{4}{*}{ Hoja } & Forma lámina & Cordada & Ovada & Cordada & Ovada & Ovada \\
\hline & Forma de la base & Subcordada & Cuneada & Cordada & Cordada & Cuneada \\
\hline & Forma del ápice & $\begin{array}{l}\text { Ampliamente } \\
\text { agudo }\end{array}$ & $\begin{array}{l}\text { Estrechamente } \\
\text { agudo }\end{array}$ & $\begin{array}{l}\text { Medianamente } \\
\text { agudo }\end{array}$ & $\begin{array}{l}\text { Ampliamente } \\
\text { agudo }\end{array}$ & $\begin{array}{l}\text { Estrechamente } \\
\text { agudo }\end{array}$ \\
\hline & $\begin{array}{l}\text { Color principal } \\
\text { lámina }\end{array}$ & Verde & Verde amarillo & Verde amarillento & Verde & Verde amarillo \\
\hline \multirow{2}{*}{ Flor } & Color botón floral & Rojo púrpura & Pardo amarillento & Amarillo & Rojo púrpura & Rojo púrpura \\
\hline & Color de Perigonio & Púrpura & Blanco grisáceo & Blanco & Púrpura & Purpura claro \\
\hline \multirow{3}{*}{ Raíz } & $\begin{array}{l}\text { Color pulpa raíz } \\
\text { tuberosa }\end{array}$ & Blanco & Blanco & Blanco & $\begin{array}{l}\text { Amarillo } \\
\text { anaranjado }\end{array}$ & Blanco \\
\hline & $\begin{array}{l}\text { Color externo raíz } \\
\text { tuberosa }\end{array}$ & $\begin{array}{l}\text { Gris anaranjado } \\
\text { claro }\end{array}$ & $\begin{array}{l}\text { Gris anaranjado } \\
\text { claro }\end{array}$ & Pardo amarillento & Gris anaranjado & Pardo amarillento \\
\hline & Entradas & $\begin{array}{l}012 ; 013,045 ; 043 ; \\
040 ; 038 ; 030 ; 029 ; \\
026 ; 024 ; 028 ; 017 ; \\
015 ; 031 ; 037 ; 025 ; 014\end{array}$ & $\begin{array}{l}001 ; 002 ; 003 ; 005 ; \\
006 ; 007 ; 008 ; 009 \\
4^{036 ; 039 ; 010 ; 011 ; 019}\end{array}$ & $\begin{array}{l}016 ; 048,020 ; 021 ; \\
9^{046,023 ; 027 ; 047}\end{array}$ & 042 & 044 \\
\hline
\end{tabular}


Tabla 5. Contribución relativa de los componentes principales de la variación total de la colección de mauka, Mirabilis expansa, de la Universidad Nacional de Cajamarca.

\begin{tabular}{ccc}
\hline $\begin{array}{c}\text { Componente } \\
\text { Principal }\end{array}$ & $\begin{array}{c}\text { Porcentaje de } \\
\text { variación }\end{array}$ & $\begin{array}{c}\text { Porcentaje de } \\
\text { variación acumulado }\end{array}$ \\
\hline 1 & 60,62 & 60,62 \\
2 & 31,56 & 92,18 \\
3 & 5,27 & 97,45 \\
4 & 2,53 & 99,98 \\
\hline
\end{tabular}

La Tabla 5 muestra la contribución relativa de los componentes principales a la variación total. Los cuatro primeros componentes principales contribuyen al $100 \%$ de la variación de los 17 caracteres, para discriminar las 40 entradas en estudio. El primer componente explica el $60,6 \%$ de la variación entre entradas y está asociado a cinco caracteres de hoja, cuatro de flor, tres de tallo, dos de raíz, y uno de follaje. Todos con un alto coeficiente de correlación que varía de 0,65 (ápice de lámina) hasta 0,99 (color predominante del haz de la hoja y color de la corteza de la raíz sin piel). Junto a los últimos mencionados, los caracteres de flor son también los que más contribuyen al primer componente.

El 31,5\% de la variación total se atribuye al segundo componente y los caracteres que más aportan a éste son el color de las brácteas del involucro y el color de la pulpa de la raíz reservante. Ambos, con un alto coeficiente de correlación (0,91). Asimismo, los caracteres foliares (forma de lámina y ápice de lámina) fueron los que más contribuyeron al tercer componente principal, lo que se evidencia por sus altos coeficientes (Tabla 6).

Tabla 6. Proporción de la varianza explicada, componentes principales y coeficientes, en la discriminación morfológica de la colección de mauka, Mirabilis expansa, de la Universidad Nacional de Cajamarca.

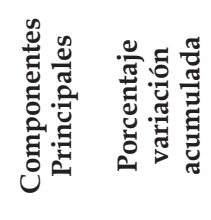

Caracteres y coeficientes

Color predominante del haz de la hoja $(0,9955)$ Color de la corteza de raíz al raspado $(0,9955)$

Color de botón floral $(0,9916)$

Color de Perigonio $(0,9916)$

Color Estigma $(0,9916)$

Color venas de lóbulos del perigonio $(0,9900)$

Base de lámina $(0,9155)$

CP1 60,62 Color principal del tallo aéreo $(0,9130)$

Color secundario del haz de la hoja $(0,9034)$

Color secundario del tallo aéreo $(0,8585)$

Color de follaje $(0,7883)$

Color de tallo subterráneo $(0,7697)$

Color externo de la corteza de la RR $(0,7331)$

Forma de lámina $(-0,6976)$

Apice de lámina $(0,6527)$

Color de las brácteas del involucro $(0,9126)$

CP2 31,56 Color de la pulpa de la raíz reservante $(0,9126)$

Color externo de la corteza de la RR $(0,5035)$

Forma de lámina $(-0,6972)$

CP3 5,27 Ápice de lámina(-0,6965)

Color de follaje $(0,5814)$
Variabilidad morfológica y distribución geográfica de los morfotipos. La conformación de los grupos o morfotipos muestra relación con la distribución geográfica de las entradas. Así, el grupo M-II está constituido en su mayoría por material procedente de la provincia de Sánchez Carrión (La Libertad), y los caracteres principales que lo discriminan son el color verde oscuro del follaje, la forma cuneada de la base de la lámina y el color blanco grisáceo del perigonio. El grupo IV (entrada 42), procedente de Puno es diferente del resto de material evaluado. Particularmente, en los caracteres de raíz y tallo. Este morfotipo no se encuentra en el norte peruano y se infiere que es endémico del sur peruano.

Como se aprecia en la Figura 2, el mayor número de morfotipos (de 2 a 4, cuadrícula roja) se concentra en las provincias de Hualgayoc, San Miguel, Chota y Cajamarca en los que predominan los morfotipos M-I, M-III.

Todos los morfotipos ocupan el rango altitudinal propio de la región Quechua, desde los $2300 \mathrm{~m}$ de altitud (Namora, Cajamarca), hasta los $3400 \mathrm{~m}$ (Huayllapampa, Cajamarca). La entrada 42 (M-IV) fue colectado a $3000 \mathrm{~m}$, Limbani (Puno). Las colectas fueron realizadas dentro de los límites latitudinales: $6^{\circ} 23^{\prime}$ y $7^{\circ} 51^{\prime} S$ en el norte, hasta los $14^{\circ} 6^{\prime} S$ en el sur del Perú. El $92 \%$ de las entradas proceden de cuencas que tributan a la vertiente del Atlántico.

Evaluación agronómica. La información que se presenta en este aspecto es preliminar, pero permite hacer algunas apreciaciones importantes. Existe alta variación en los principales componentes del rendimiento, entre entradas del mismo morfotipo, entre morfotipos (Tabla 7). Los los morfotipos de mayor rendimiento son el I, II y III ( 46 a 76 t.ha $\mathrm{a}^{-1}$ ) y las entradas promisorias, en orden de importancia son 15, 16, 25, 28, 24, 17.

Los componentes del rendimiento mostraron variaciones importantes en las tres campañas. Así, el peso de raíces por planta varió de 0,6 a 3,6 kg.ha-1. El número de raíces por planta varió

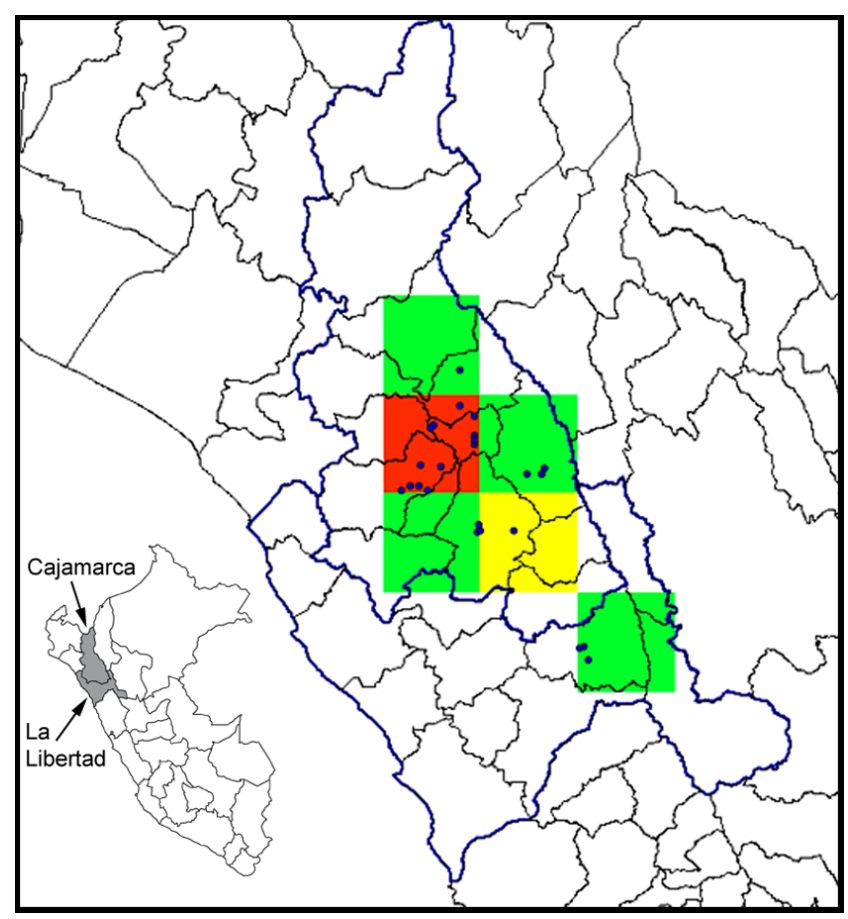

Figura 2. Distribución geográfica de los morfotipos de mauka, Mirabilis expansa, del norte peruano. 
Tabla 7. Valores de los componentes del rendimiento de 40 entradas de mauka (Mirabilis expansa), agrupadas por morfotipos (promedios de tres campañas agrícolas, muestra: 15 plantas por entrada - 5 por campaña).

\begin{tabular}{|c|c|c|c|c|c|c|}
\hline Entrada & Altura de planta $(\mathrm{cm})$ & $\mathrm{N}^{\circ}$ ráices/planta & Largo raíz (cm) & Peso raíz (kg/plta) & Rdto (kg/ha) & Peso follaje (kg/plta) \\
\hline \multicolumn{7}{|l|}{ Morfotipo I } \\
\hline 12 & 70,8 & 15,9 & 11,2 & 1,8 & 37,8 & 1,7 \\
\hline 13 & 68,1 & 17,3 & 13,4 & 1,4 & 30,7 & 1,9 \\
\hline 14 & 74,8 & 13,5 & 14,7 & 1,4 & 30,7 & 2,1 \\
\hline 15 & 79,5 & 21,7 & 16,3 & 3,6 & 76,4 & 2,8 \\
\hline 17 & 90,5 & 19,7 & 15,6 & 2,2 & 45,8 & 2 \\
\hline 24 & 86,4 & 18,8 & 12,4 & 2,2 & 47 & 1,5 \\
\hline 25 & 88,3 & 20,6 & 15,5 & 3 & 63,4 & 2,4 \\
\hline 26 & 74,7 & 21,1 & 14,2 & 1,7 & 35,7 & 1,2 \\
\hline 28 & 79,1 & 20,6 & 16,2 & 2,7 & 57,7 & 1,9 \\
\hline 29 & 66,2 & 19 & 16,1 & 1,5 & 33 & 1,1 \\
\hline 30 & 75,7 & 18 & 14,7 & 1,8 & 38,1 & 1,7 \\
\hline 31 & 72,5 & 16,2 & 11,6 & 1,5 & 33,1 & 1 \\
\hline 37 & 51,5 & 15,3 & 11,6 & 0,7 & 15,9 & 1,3 \\
\hline 38 & 66,3 & 10,8 & 11,9 & 1,5 & 31,5 & 0,6 \\
\hline 40 & 67,3 & 14,1 & 18,1 & 1,6 & 35 & 1,7 \\
\hline 43 & 31,5 & 10 & 14,6 & 1,7 & 35,3 & 1,1 \\
\hline 45 & 102,9 & 8 & 12,91 & 0,8 & 17,6 & 1,6 \\
\hline Prom & 73,3 & 16,5 & 14,2 & 1,8 & 39,1 & 1,6 \\
\hline DS & 15,8 & 4,1 & 2 & 0,7 & 15,3 & 0,5 \\
\hline \multicolumn{7}{|l|}{ Morfotipo II } \\
\hline 1 & 84,5 & 12,6 & 18 & 1,1 & 23,9 & 1,4 \\
\hline 2 & 85,2 & 15,2 & 15,2 & 0,9 & 20,3 & 1,8 \\
\hline 3 & 73,3 & 10,5 & 13,4 & 0,6 & 13,1 & 1,7 \\
\hline 5 & 76,6 & 15,1 & 15,1 & 0,9 & 18,7 & 1,3 \\
\hline 6 & 74,5 & 16 & 16,2 & 0,9 & 19,7 & 2 \\
\hline 7 & 79,1 & 11,6 & 14,9 & 0,7 & 14,5 & 1,3 \\
\hline 8 & 79,7 & 15,6 & 16,2 & 0,6 & 13,3 & 1,5 \\
\hline 9 & 68,6 & 15,7 & 16,9 & 1,2 & 25 & 1,3 \\
\hline 10 & 72,3 & 15,4 & 18 & 1,3 & 27,3 & 2,2 \\
\hline 11 & 75,1 & 18,6 & 15,9 & 1 & 21 & 2,3 \\
\hline 19 & 94,4 & 19 & 17,4 & 1,6 & 33,3 & 1,6 \\
\hline 36 & 79,6 & 12,6 & 10,7 & 0,8 & 17,4 & 0,4 \\
\hline 39 & 65,7 & 9,7 & 14,9 & 0,9 & 19 & 0,5 \\
\hline Prom & 77,6 & 14,4 & 15,6 & 0,9 & 20,5 & 1,5 \\
\hline DS & 7,5 & 2,8 & 1,9 & 0,2 & 5,7 & 0,5 \\
\hline \multicolumn{7}{|l|}{ Morfotipo III } \\
\hline 16 & 62,5 & 17,7 & 14,5 & 3 & 63,9 & 2,5 \\
\hline 20 & 96,3 & 15,2 & 13,4 & 2 & 42,9 & 2,3 \\
\hline 21 & 83,3 & 11,8 & 13,5 & 1,6 & 34,8 & 1,1 \\
\hline 23 & 88,6 & 18 & 13,7 & 1,3 & 27,9 & 1,7 \\
\hline 27 & 60,2 & 17,2 & 16 & 2,2 & 47,1 & 1,7 \\
\hline 46 & 86 & 4 & 10,7 & 0,6 & 12,4 & 2,4 \\
\hline 47 & 75 & 13 & 13,1 & 1,2 & 24,9 & 1 \\
\hline 48 & 98 & 5 & 8,4 & 0,8 & 16,6 & 0,8 \\
\hline Prom & 81,2 & 12,7 & 12,9 & 1,6 & 33,8 & 1,7 \\
\hline DS & 14,2 & 5,5 & 2,3 & 0,8 & 17 & 0,6 \\
\hline \multicolumn{7}{|l|}{ Morfotipo IV } \\
\hline 42 & 47 & 10,5 & 13 & 1,4 & 29,1 & 0,6 \\
\hline \multicolumn{7}{|l|}{ Morfotipo V } \\
\hline 44 & 59,3 & 14 & 12,9 & 1,6 & 33,2 & 1,7 \\
\hline
\end{tabular}

de 4 a 22, la altura de planta varió de 31,5 a $103 \mathrm{~cm}$. Además de las raíces, las plantas, producen una cantidad de follaje (útil como forraje) que varió entre 0,26 a 3,9 kg.planta ${ }^{-1}(1,4 \mathrm{~kg}$. planta $^{-1}$, en promedio).

\section{Discusión}

Se confirma la escasa variabilidad de $M$. expansa al estado cultivado y a la vez que, en Perú se encuentra la mayor variabilidad. La caracterización morfológica y molecular de Tapia et al. (2004) en la colección ecuatoriana discriminó tres morfotipos (de los cuales, uno es el morfotipo I de la colección del presente estudio). Por otro lado, en el germoplasma boliviano se ha observado dos morfotipos, blanco y amarillo (Rea 2004). También, los resultados confirman que en el norte peruano, específicamente en Cajamarca, se encuentra la mayor variabilidad morfológica.

Los descriptores usados resultan eficientes para identificar morfotipos, facilitan el trabajo de campo y permiten inferir que el morfotipo $\mathrm{V}$ es un híbrido intraespecífico, producto de cruzamiento natural entre materiales de los morfotipos I y II. Lo anterior se explica porque las maukas cultivadas se cruzan fácilmente y la especie registra entre 6 y $25 \%$ de alogamia (Valderrama \& Seminario 2001). Sin embargo, esta hipótesis debe someterse a prueba mediante cruzamiento recíprocos entre ambos grupos.

Los resultados concuerdan con los obtenidos por Chía et al. (2006), respecto a la separación de la entrada 42 (procedente de Puno), en un grupo aparte. Sin embargo, en el resto de resultados difieren ampliamente y no son comparables por las siguientes razones:

En primer lugar, nosotros trabajamos con 40 entradas cultivadas, Chía et al. (2006) trabajaron con 34 entradas cultivadas de la misma colección. Dos de estas entradas ya no están en la colección, por eso no se incluyen en el presente trabajo. En segundo lugar, el agrupamiento morfológico que presentan los autores mencionados (Tabla 2, p.85) lo hicieron con descriptores 
morfológicos no estandarizados, de modo que no coincide con el agrupamiento actual (Tabla 4), realizado con 17 descriptores morfológicos probados y estandarizados. En tercer lugar, si bien, Chía et al. (2006) establecieron (coeficiente de 0,85) que el grupo 1 está formado por entradas que pertenecen a los morfotipos I, II, III y V (entradas con características morfológicas disímiles) (Tabla 4), a la vez, el grupo 4, está formado por entradas pertenecientes a los morfotipos I, II y III, lo cual aparentemente es poco congruente. Asimismo, considerando que el grupo 2 está formado sólo por entradas del morfotipo I, parece poco congruente que entradas de este mismo morfotipo estén también en los grupos 1 y 4 de estos autores.

En investigaciones precedentes es frecuente la alta concordancia entre el agrupamiento morfológico (si se realiza con descriptores estandarizados) y el agrupamiento molecular. Así lo indican Tapia et al. (2004) en maukas ecuatorianas. También, Blas (2005), en arracacha (también tuberosa), encontró hasta 97\% de concordancia entre el agrupamiento morfológico y el molecular, con variaciones, según la técnica empleada (RAPD, AFLP). Por lo tanto, es necesario hacer un nuevo análisis molecular para la colección de mauka, para confirmar los resultados y poderlos comparar con el agrupamiento morfológico, aquí descrito.

Es importante que los cuatro primeros $\mathrm{CP}$ contribuyan con el $100 \%$ de la variación de los 17 caracteres y que el primer CP explica el 60,6\% de la variación entre entradas. Este CP está asociado características altamente discrimantes e identificables en campo (cinco de hoja, cuatro de flor, tres de tallo, dos de raíz, y uno de follaje) de mucha utilidad para trabajos futuros (Hidalgo 2003).

Los morfotipos presentan patrones de distribución, que podrían indicar que han evolucionado en condiciones locales y no han sido objeto de movimientos interregionales en épocas recientes. Así, los morfotipos I y III, se distribuyen ampliamente en la región Cajamarca. En esta región se han registrado la mayor cantidad de nombres comunes para la especie al estado cultivado (Seminario 2004). El grupo M-II, probablemente es endémico de la Región La Libertad, con extensión hacia el sur (Región Ancash). El morfotipo IV está localizado en la región Puno y podría tratarse del mismo morfotipo que describen Vallenas (1995) y Rea (1992) en esta esta región.

La conclusión provisional de que la mayor variabilidad se encuentra en la Región Cajamarca, requiere confirmación a través de exploraciones exhaustivas en Sihuas, Carhuaz y otras provincias de Ancash y en las zonas altas de Amazonas. También es conveniente realizar exploraciones en Huánuco, Junín, Ayacucho, Cusco, Apurimac y Puno.

El rendimiento promedio de raíces $\left(31 \mathrm{t}^{\mathrm{h} \mathrm{ha}^{-1}}\right)$ y su alta variación (12 a $76 \mathrm{t} \mathrm{ha}^{-1}$ ), así como la variación de sus componentes (altura de planta, número y peso de raíces), confieren al germoplasma alto valor para el mejoramiento y se requiere otras investigaciones para explorar su sobre su potencial. Anteriormente se informó de rendimientos de 45 a 137 t.ha ${ }^{1}$ (Seminario 2004), en materiales de la misma región. Tapia et al. (2004) informan que en maukas ecuatorianas se obtuvieron rendimientos de 40 t.ha ${ }^{-1}$. Por ser materiales recolectados en diferentes nichos y en cultivos asociados, se espera que tengan respuesta variable a condiciones ex situ, bajo nuevas presiones ambientales -suelo, clima, monocultivo- (Holle 1991).

\section{Agradecimientos}

A la Facultad de Ciencias Agrarias de la UNC por el apoyo financiero para la realización de la investigación. A Griselda Lay de la Biblioteca del Centro Internacional de la Papa, por su apoyo con artículos sobre mauka. A Segundo Cusquisiban por el apoyo en el trabajo de campo.

\section{Literatura citada}

Bazán M., J.I. López \& W. Pajares. 1996. Potencial forrajero del chago (Mirabilis expansa) en la alimentación de conejos (Oryctolagus cuniculus). Tesis Ing. Zoot. Cajamarca, Facultad de Zootecnia, Universidad Nacional de Cajamarca. 93 p.

Blas R. 2005. Diversity of Arracacia species in Peru. Dissertation originale présentée en vue de l'obtention du grade de docteur en Sciences Agronomiques et Ingénierie Biologique. Faculte Universitaire des Sciences Agronomiques de Gembloux. 170 p.

Chia J., C. López, R. Blas, J. Seminario, R. Mansilla \& J. Baudoin. 2006. Diversidad genética molecular de Mirabilis expansa mediante RAPD. En: Ecología Aplicada, 5(1,2), 2006.

Crisci J.V. \& M.F. López. 1983 Introducción a la teoría y práctica de la taxonomía numérica. Serie de Biología. Monografía No. 26. Secretaría General de la O.E.A. Programa Regional de Desarrollo Científico y Tecnológico, Washington, D.C. $132 \mathrm{p}$.

D.S. 043-2006-AG. 2006. Aprueban categorización de especies amenazadas de flora Silvestre. El Peruano, Lima PE, jul. 13 (normas legales): 323527-323539.

FAO. 2009. Informe nacional sobre el estado de los recursos fitogeneticos para la agricultura y la alimentación - Bolivia. Proyecto: Fortalecimiento de los bancos de germoplasma vegetal del sistema nacional de recursos genéticos para la agricultura y la alimentación, Bolivia. Disponible en: http://www.fao.org/docrep/013/i1500e/Bolivia.pdf

Franco S. \& J. Uceda. 1996. El chago o yuca inca (Mirabilis expansa) una raíz andina en peligro de extinción. Informe Técnico. Estación Experimental Agropecuaria y Forestal Baños del Inca, INIAA, $\mathrm{N}^{\circ} 1$. Cajamarca.

Hidalgo R. 2003. Variabilidad genética y caracterización de especies vegetales, En: Franco, T. \& Hidalgo, R. (eds). Análisis estadístico de datos de caracterización morfológica de Recursos fitogenéticos. Boletín técnico $\mathrm{N}^{\circ} 8$, Instituto Internacional de Recursos Fitogenéticos (IPGRI), Cali, Colombia. pp: 2-49.

Holle M. 1991. Exploración y recolección sistemática de plantas cultivadas en la zona andina para el desarrollo de recursos fitogenéticos. En: Castillo, R.: Estrella, J. y Tapia, C. (eds). Técnicas para el manejo y uso de los recursos fitogenéticos. INIAP, Quito, Ecuador. pp: 52-63.

Klásková T. \& C. Fernandéz. 2011. Mauka - a promise for fighting with the unbalanced nutrition of high andean regions. In: Agricultura Tropica et Subtropica 44 (1) 2011. Pp: 37-40. Institute of Tropics and Subtropics, Czech University of Life Sciences, Prague, Czech Republic.

NRC (National Research Council). 1989. Lost crops of the Incas: Little-known plants of the Andes with promise for worldwide cultivation. Washington, DC. (USA). National Academy Press. 415 p.

Park S-W., N.M. Stevens \& J.M. Vivanco. 2002. Enzymatic specificity of three ribosome-inactivating proteins against fungal ribosomes, and correlation with antifungal activity. Planta 216: $227-234$.

Rea J. 1992. Raíces Andinas. En: J.E. Hernández y J. León (eds.) Cultivos marginados, otra perspectiva de 1492. Colección FAO. Producción y protección vegetal $\mathrm{N}^{\circ} 26$. Roma, Italia. Pp: 163-177. 
Rea J. 2004. Conservación y manejo in situ de recursos fitogenéticos agrícolas en Bolivia (3). En: J. Seminario (ed.) Raíces Andinas: Contribuciones al conocimiento y a la capacitación. Serie: Conservación y uso de la biodiversidad de raíces y tubérculos andinos: Una década de investigación para el desarrollo (1993 -2003) $\mathrm{N}^{\circ}$ 6. Universidad Nacional de Cajamarca, Centro Internacional de la Papa, Agencia Suiza para el Desarrollo y la Cooperación. Lima, Perú. pp: 49-64.

Seminario J. 2004. Aspectos etnobotánicos y productivos del chago, miso o mauka (Mirabilis expansa (R.\&P.) Standley (26). En: J. Seminario (ed.) Raíces Andinas: Contribuciones al conocimiento y a la capacitación. Serie: Conservación y uso de la biodiversidad de raíces y tubérculos andinos: Una década de investigación para el desarrollo (1993 -2003) $\mathrm{N}^{\circ}$ 6. Universidad Nacional de Cajamarca, Centro Internacional de la Papa, Agencia Suiza para el Desarrollo y la Cooperación. Lima, Perú. pp: 367-376.

Tapia, C., J. Estrella., A. Monteros, F. Valverde, M. Nieto \& J. Córdova. 2004. Manejo y Conservación de RTAs in situ en fincas de agricultores y ex situ en el Banco de Germoplasma de INIAP. En: Barrera, V., Tapia, C. y Monteros A. (eds.). Raíces y Tubérculos Andinos: Alternativas para la conservación y uso sostenible en el Ecuador. Serie: Conservación y uso de la biodiversidad de raíces y tubérculos andinos: Una década de investigación para el desarrollo (1993-2003). INIAP, CIP, COSUDE. Quito, Ecuador Lima, Perú. pp. 31 - 74.
Valderrama M. \& J. Seminario. 2001. Biología floral de cuatro cultivares y tres parientes silvestres de mauka, Mirabilis expansa (R.\&P.) Standley. En ARNALDOA 8(1): 71-86. UPAO, Trujillo, Perú.

Vallenas M. 1995. Vigencia del cultivo de mauka (Mirabilis expansa) en Puno, Perú. En: Resúmenes del primer Congreso peruano de Cultivos Andinos "Oscar Blanco Galdós". 11 -16 setiembre, Huamanga, Ayacucho. pp: 72-73.

Vepachedu R., H.P. Bais \& J.M. Vivanco. 2003. Molecular characterization and post-transcripcional regulation of ME1 , a type-I ribosome-inactiving protein from Mirabilis expansa. Planta 217: 498-506.

Vepachedu R., S.W. Park, N. Sharma \& J.M. Vivanco. 2005. Bacterial expression and enzymatic activity analysis of ME1, a ribosome-inactiving protein from Mirabilis expansa. Protein Expression and Purification 40: 142-151.

Vivanco J.M., B.J. Savary \& H.E. Flores. 1999. Characterization of two novel type 1 ribosome-inactivating proteins from the storage roots of the andean crop Mirabilis expansa. Plant Physiology 119: 1447-1456.

Vivanco J.M. \& H.E. Flores. 2000. Biosynthesis of ribosomeinactivating proteins from callus and cell suspensión cultures of Mirabilis expansa (Ruiz y Pavon). Plant Cell reports 19: 1033-1039. 